\title{
Associação entre cobertura por serviços de saneamento e indicadores epidemiológicos nos países da América Latina: estudo com dados secundários
}

\author{
Júlio César Teixeira, ${ }^{1}$ Maria Helena Rodrigues Gomes ${ }^{1}$ \\ e Janaina Azevedo de Souza ${ }^{1}$
}

Como citar Teixeira JC, Gomes MHR, de Souza JA. Associação entre cobertura por serviços de saneamento e indicadores epidemiológicos nos países da América Latina: estudo com dados secundários. Rev Panam Salud Publica. 2012:32(6):419-25.

RESUMO Objetivo. Estudar a associação entre cobertura por serviços de saneamento básico e indicadores epidemiológicos nos países da América Latina, utilizando dados secundários da Organização Pan-Americana da Saúde (OPAS) relativos ao período de 2005 a 2010.

Métodos. Realizou-se um estudo ecológico incluindo dados de 21 países da América Latina, com população total estimada de 596 milhões de habitantes. Foram analisadas: mortalidade infantil em menores de 1 ano em 2008, mortalidade em menores de 5 anos em 2008 e mortalidade por enfermidades diarreicas agudas em menores de 5 anos em 2008. Esses indicadores foram analisados por meio de sua correlação "um a um" com indicadores de cinco classes de fatores de risco (demográficos, socioeconômicos, de saúde infantil, de gasto público com saúde e de cobertura por serviços de saúde e saneamento).

Resultados. Os valores médios para a região foram de 18,2 óbitos de crianças menores de 1 ano por 1000 nascidos vivos, 23,5 óbitos de menores de 5 anos por 1000 nascidos vivos e mortalidade de 5,8\% por enfermidades diarreicas agudas em menores de 5 anos. Quanto maior a cobertura populacional por serviços de esgotamento sanitário e por sistemas de abastecimento de água, menor a mortalidade infantil e menor a taxa de mortalidade de menores de 5 anos de idade. Quanto maior a taxa de crescimento anual da população, maior a mortalidade por enfermidades diarreicas agudas em menores de 5 anos.

Conclusões. A melhoria da saúde pública na América Latina depende da ampliação do acesso aos serviços públicos de abastecimento de água e de esgotamento sanitário.

Palavras-chave Saúde pública; saneamento; água; águas residuárias; América Latina.

O saneamento básico é um dos fatores determinantes da saúde. Sendo assim, há um reconhecimento científico quanto à relação de causalidade entre

\footnotetext{
1 Universidade Federal de Juiz de Fora (UFJF), Faculdade de Engenharia, Departamento de Engenharia Sanitária e Ambiental, Juiz de Fora (MG), Brasil. Correspondência: Júlio César Teixeira, juliotei@terra.com.br
}

condições inadequadas de saneamento básico - abastecimento de água potável, esgotamento sanitário, limpeza urbana, manejo de resíduos sólidos e drenagem - e o quadro de saúde pública existente em um determinado local, em um determinado momento. Heller (1), mediante extensa revisão da literatura sobre a relação entre saneamento básico e saúde pú- blica, consolidou o entendimento de que há melhora dos indicadores de saúde com a ampliação da cobertura por serviços de saneamento básico.

Dessa forma, a infraestrutura sanitária deficiente desempenha uma interface com a situação de saúde e com as condições de vida das populações dos países em desenvolvimento, onde as 
doenças infecciosas continuam sendo uma importante causa de morbidade e mortalidade. A prevalência dessas doenças constitui um forte indicativo de fragilidade dos sistemas públicos de saneamento (2).

No entanto, a mensuração das condições de vida e saúde persiste como um desafio. A saúde deve ser pensada como resultado das relações entre variáveis ambientais, sociais e econômicas que pressionam as condições e a qualidade de vida. Logo, na análise da situação da saúde, indicadores de desenvolvimento humano assumem uma importância fundamental, pois documentam as condições de vida da população e dimensionam o espaço social em que ocorrem as mudanças no estado de saúde (3) apesar das limitações desses indicadores, que podem, por exemplo, excluir parcelas significativas da população exposta ao risco de adoecer.

Estima-se que 471 milhões de pessoas vivam em cidades na América Latina, o que equivalia, em 2010, a $79 \%$ do total de uma população estimada em 597 milhões de habitantes. Essa distribuição faz da América Latina a região mais urbanizada do planeta, com um processo de urbanização que pode ser considerado exitoso, já que trouxe riqueza, aumento da expectativa de vida e acesso a serviços públicos básicos para milhões de pessoas (4). Por outro lado, o processo de urbanização latino-americano se desenvolveu com grandes diferenças entre ricos e pobres, criando problemas nas cidades, com informalidade na moradia e no emprego. Apesar de a América Latina ser a região em desenvolvimento mais próxima de alcançar antecipadamente o Objetivo de Desenvolvimento do Milênio que diz respeito ao acesso à água potável e ao esgotamento sanitário (5), sabe-se que, quanto menor a renda per capita e o número médio de anos de estudo, mais vulnerável está a população à exclusão sanitária.

Atualmente, 80 milhões de latino-americanos ainda carecem de acesso à água potável e mais de 100 milhões não dispõem de serviços adequados de esgotamento sanitário (6). Ainda, somente 14\% das águas residuárias na América Latina são tratadas; $40 \%$ do total de resíduos sólidos não são dispostos adequadamente, contaminando o solo e os mananciais de água (7). Cerca de 23 crianças em 1000 nascidos vivos morrem antes de comple- tar 5 anos de idade, o que corresponde a 200 mil mortes por ano. Dessas crianças, 20 mil morrem a cada ano como resultado de doenças diarreicas agudas que poderiam ser evitadas pelo acesso a condições de higiene adequadas e a infraestrutura de esgotamento sanitário e de abastecimento com água potável (8).

Nesse contexto, o objetivo do presente trabalho foi investigar a associação entre indicadores epidemiológicos — taxa de mortalidade infantil, mortalidade em menores de 5 anos e mortalidade por enfermidades diarreicas agudas em menores de 5 anos - e condições de saneamento básico - cobertura populacional por sistemas de abastecimento de água e por sistemas de esgotamento sanitário nos países da América Latina, utilizando dados secundários atualizados e disponibilizados recentemente pela Organização Pan-Americana de Saúde (OPAS).

\section{MATERIAIS E MÉTODOS}

Realizou-se um estudo ecológico, que pode ser melhor compreendido como o estudo epidemiológico que utiliza agregados populacionais como unidade de análise. Tal delineamento é útil para detectar associações entre exposições indicadores de condições de vida - e indicadores epidemiológicos - ocorrência de morbimortalidade. Entretanto, o delineamento ecológico apresenta como principais limitações o pouco desenvolvimento das técnicas de análise de dados e a vulnerabilidade à falácia ecológica (9). Um exemplo da falácia ecológica é supor que os indicadores de uma determina área se referem à população total daquela área, quando na realidade os dados refletem uma média da variação de diferentes subgrupos com características próprias que vivem naquele local. Portanto, é nesse contexto que devem ser interpretados os resultados deste estudo.

$\mathrm{O}$ universo da pesquisa foi composto pelos 21 países integrantes da América Latina: Argentina, Belize, Bolívia, Brasil, Chile, Colômbia, Costa Rica, Cuba, Equador, El Salvador, Guatemala, Honduras, México, Nicarágua, Panamá, Paraguai, Peru, Porto Rico, República Dominicana, Uruguai e Venezuela. A população total desse grupo de países é estimada em 597 milhões de habitantes. A taxa estimada de crescimento da população da região para as três primeiras décadas do século XXI é de $1,1 \%$ ao ano (10).
A base de informações foi composta por dados secundários disponibilizados pela OPAS para o período de 2005 a 2010 (11). Foi verificada a ausência de correlação entre os indicadores de saúde (variáveis dependentes), o que garante a adequação do modelo utilizado. Foram analisadas as seguintes variáveis dependentes:

- Taxa de mortalidade infantil em 2008: número de óbitos de crianças menores de 1 ano de idade por 1000 nascidos vivos.

- Mortalidade em menores de 5 anos de idade em 2008: número de óbitos de crianças menores de 5 anos de idade por 1000 nascidos vivos.

- Mortalidade por enfermidades diarreicas agudas em menores de 5 anos de idade em 2008: \% do total de óbitos em crianças menores de 5 anos causados por enfermidade infecciosa intestinal.

Cada um dos três indicadores de saúde estudado foi analisado por meio de sua correlação "um a um" com vários outros indicadores, divididos em cinco classes de fatores de risco (variáveis independentes), conforme descrito a seguir.

\section{Indicadores demográficos}

- Taxa de crescimento anual da população em 2009/2010 (\% de incremento populacional).

- Proporção de urbanização em 2010 (\% da população urbana).

- Taxa de fecundidade total em 2010 (número médio de nascidos vivos por mulher).

- Taxa bruta padronizada de natalidade em 2010 (número de nascidos vivos por 1000 habitantes).

- Esperança de vida ao nascer em 2010 (número de anos de vida esperados).

\section{Indicadores socioeconômicos}

- Taxa de alfabetização em 2007/2008 (\% de alfabetizados na população de 15 anos ou mais de idade).

- Produto interno bruto (PIB) per capita em 2008 (dólares per capita).

- Razão de renda em 2006/2007 (número de vezes pelas quais a renda dos $20 \%$ mais ricos supera a dos $20 \%$ mais pobres em um país).

- Proporção da população abaixo da linha internacional de pobreza de 2005 
a 2008 (\% da população com renda per capita diária inferior a US\$2).

- Taxa de desemprego de 2005 a 2008 (\% da população economicamente ativa que se encontrava sem trabalho).

\section{Indicadores de saúde infantil}

- Proporção de nascidos vivos de baixo peso ao nascer de 2005 a 2008 (\% de nascidos com peso inferior a $2500 \mathrm{~g}$ ).

- Prevalência anual de déficit nutricional moderado e grave em crianças menores de 5 anos de 2005 a 2008 (\%).

- Prevalência de aleitamento materno exclusivo até 120 dias no período de 2005 a 2008 (\% dos lactentes).

\section{Indicadores de gasto público} com saúde

- Gasto público anual com saúde como proporção do PIB em 2008 (\% do PIB).

\section{Indicadores de cobertura por serviços de saúde e de saneamento}

- Número de atendimentos ambulatoriais de 2005 a 2008 (consultas/1 000 habitantes).

- Número de internações hospitalares de 2005 a 2008 (internações / 1000 habitantes).

- Proporção de gestantes com acompanhamento pré-natal de 2005 a 2008 (\% em relação ao total de gestantes).

- Proporção da população imunizada contra difteria, coqueluche e tétano em 2008 (\% de menores de 1 ano imunizados).

- Cobertura por sistemas de abastecimento de água em 2008 (\% da população urbana).

- Cobertura por sistemas de esgotamento sanitário em 2008 (\% da população urbana).

\section{Análise dos dados}

Os dados foram analisados por meio de regressão linear com medidas de associação com o coeficiente de determinação $R^{2}$, seguido de seu teste de significância. $O$ coeficiente de determinação $R^{2}$ varia no intervalo de 0 a 1 . Quanto maior o seu valor, mais explicativo será o modelo proposto. Além disso, foi realizada uma análise em etapas, de forma a permitir a determinação progressiva das exposições efetivamente associadas aos indicadores de saúde estudados. Outros estudos $(12,13)$ adotaram essa sistemática, apresentando resultados satisfatórios quanto à consistência e ao rigor científico. O processo envolveu, em sequência, as seguintes atividades:

- regressão linear simples entre os indicadores epidemiológicos e cada um dos indicadores demográficos, socioeconômicos, de saúde infantil, de gasto público com saúde e de cobertura por serviços de saúde e saneamento, de modo a avaliar a relação entre as variáveis dependentes e as variáveis independentes e pré-selecionar os indica- dores a serem utilizados na regressão linear multivariada em nível de $10 \%$ de significância $(P \leq 0,10)$;

- análise de regressão linear múltipla, na qual foram identificadas as variáveis independentes estatisticamente associadas a cada indicador epidemiológico estudado em nível de significância de $5 \%(P \leq 0,05)$.

Foi utilizado o programa Statistical Package for the Social Sciences (SPSS) 15.0.

\section{RESULTADOS}

\section{Taxa de mortalidade infantil}

Inicialmente, observou-se que, para os países da América Latina, a menor taxa de mortalidade infantil foi a de Cuba, com 4,7 óbitos de crianças menores de 1 ano por 1000 nascidos vivos. A maior taxa foi a da Bolívia, com 50,0 óbitos de menores de 1 ano por 1000 nascidos vivos para o ano de 2008. O valor médio foi de 18,2 óbitos de crianças menores de 1 ano por 1000 nascidos vivos.

Ao se processar a análise de regressão linear múltipla para a taxa de mortalidade infantil, encontrou-se um coeficiente $\mathrm{R}^{2}$ ajustado de 0,700 , sendo que os indicadores que permaneceram no modelo final, com $P \leq 0,05$, foram cobertura por sistemas de esgotamento sanitário $(P<0,001)$ e gasto público anual com saúde como proporção do PIB $(P=0,007)$ (tabela 1$)$.

Os indicadores de cobertura por sistemas de esgotamento sanitário e gasto

TABELA 1. Análise de correlação linear múltipla entre taxa de mortalidade infantil, mortalidade em menores de 5 anos e mortalidade por enfermidades diarreicas agudas em menores de 5 anos e fatores de risco, América Latina, 2008

\begin{tabular}{|c|c|c|c|c|c|}
\hline $\mathrm{R}^{2 \mathrm{a}}$ & $\mathrm{R}^{\mathrm{b}}$ & Variáveis que permaneceram ${ }^{c}$ & $\beta^{\mathrm{d}}$ & $P$ valore & Sinal $^{f}$ \\
\hline \multicolumn{6}{|c|}{ Taxa de mortalidade infantil } \\
\hline \multirow[t]{2}{*}{0,700} & 0,837 & Esgotamento sanitário & $-0,657$ & $<0,001$ & - \\
\hline & & Gasto público anual com saúde como proporção do PIB & $-0,389$ & 0,007 & - \\
\hline 0,556 & 0,746 & Sistemas de abastecimento de água & $-0,646$ & $<0,001$ & - \\
\hline \multicolumn{6}{|c|}{ Mortalidade em menores de 5 anos } \\
\hline \multirow{2}{*}{0,556} & 0,746 & Sistemas de abastecimento de água & $-0,646$ & $<0,001$ & - \\
\hline & & Razão de renda & $+0,410$ & 0,013 & + \\
\hline \multicolumn{6}{|c|}{ Mortalidade por enfermidades diarreicas em menores de 5 anos } \\
\hline \multirow[t]{3}{*}{0,742} & 0,861 & Proporção de nascidos vivos com < $2500 \mathrm{~g}$ & $+0,517$ & $<0,001$ & + \\
\hline & & PIB per capita & $-0,407$ & 0,005 & - \\
\hline & & Taxa de crescimento anual da população & $+0,407$ & 0,005 & + \\
\hline
\end{tabular}

$\mathrm{R}^{2}=$ coeficiente de determinação.

${ }^{b} \mathrm{R}=$ coeficiente de correlação linear de Pearson.

c $P \leq 0,05$.

d $\beta=$ coeficiente angular da correlação linear.

e $P$ valor = significância estatística do coeficiente angular da correlação linear

Sinal $=$ sinal do coeficiente angular da correlação linear. 
FIGURA 1. Regressão linear simples entre a taxa de mortalidade infantil e cobertura por sistemas de esgotamento sanitário, América Latina, 2008

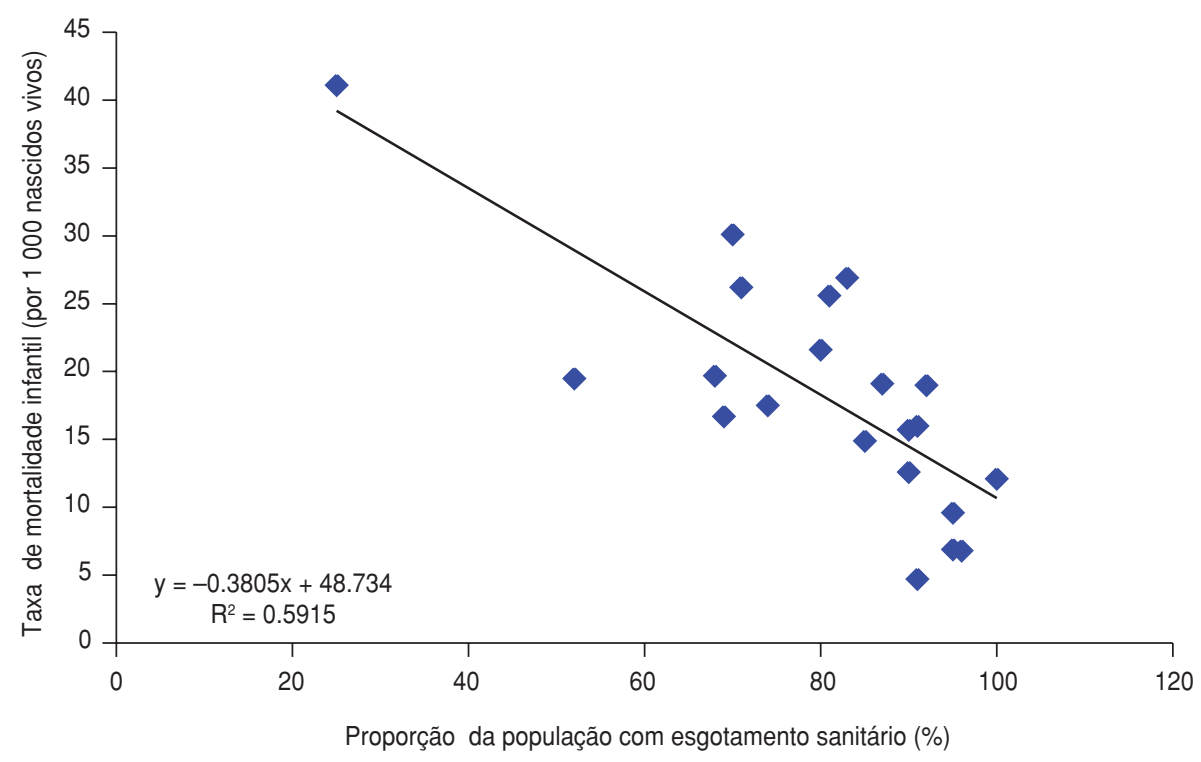

público anual com saúde como proporção do PIB apresentaram um coeficiente angular da correlação linear ( $\beta$ ) negativo, mostrando uma relação inversamente proporcional com a taxa de mortalidade infantil nos países da América Latina, conforme esperado para essa relação. $\mathrm{Na}$ figura 1, observa-se que quanto maior a cobertura populacional por serviços de esgotamento sanitário menor é a mortalidade infantil em países da América Latina.

\section{Mortalidade em menores de 5 anos de idade}

Entre os países estudados, a taxa média de mortalidade de menores de 5 anos foi de 23,5 óbitos por 1000 nascidos vivos. O menor valor foi o de Cuba, com 7,5 mortes de menores de 5 anos por 1000 nascidos vivos e o maior valor foi observado na Bolívia, com 59,5 óbitos de menores de 5 anos de idade por 1000 nascidos vivos para o ano de 2008.

Ao se processar a análise de regressão linear múltipla, encontrou-se um coeficiente $\mathrm{R}^{2}$ ajustado de 0,556 , sendo que as variáveis que permaneceram no modelo final, com $P \leq 0,05$, foram cobertura por sistemas de abastecimento de água $(P<$ $0,001)$ e razão de renda $(P=0,013)$.

$\mathrm{O}$ indicador cobertura por sistemas de abastecimento de água apresentou coeficiente $\beta$ negativo, confirmando uma relação esperada, inversamente proporcional à mortalidade de menores de 5 anos de idade. Já o indicador razão de renda apresentou coeficiente $\beta$ positivo, mostrando uma relação também esperada, diretamente proporcional à mortalidade de menores de 5 anos de idade (tabela 1). A figura 2 mostra que quanto maior a cobertura populacional por sistemas de abastecimento de água em países da América Latina menor a taxa de mortalidade de menores de 5 anos de idade na região.

\section{Mortalidade por enfermidades diarreicas agudas em menores de 5 anos}

Em 2008, a mortalidade média por enfermidades diarreicas agudas em menores de 5 anos foi de $5,8 \%$, sendo que o menor valor, de $1,0 \%$, foi observado na Costa Rica e o maior valor, de $13,1 \%$, foi observado na Guatemala.

Quando da análise de regressão linear multivariada (tabela 1), identificou-se um coeficiente $R^{2}$ ajustado de 0,742, sendo que as variáveis que permaneceram no modelo final foram proporção de nascidos vivos de baixo peso ao nascer $(P<0,001)$, PIB per capita $(P=0,005)$ e taxa de crescimento anual da população $(P<0,005)$. Os indicadores de proporção de nascidos vivos de baixo peso ao nascer e taxa de crescimento anual da população apresentaram coeficiente $\beta$ positivo, mostrando uma relação diretamente proporcional com a mortalidade por enfermidades diarreicas agudas em menores de 5 anos de idade. Já o indicador PIB per capita apresentou um valor de $\beta$ negativo, mostrando uma relação inversamente proporcional com o indicador em estudo. Todas as correlações encontradas eram esperadas (tabela 1).

A figura 3 mostra que quanto maior a taxa de crescimento anual da população em um país da América Latina maior foi a mortalidade por enfermidades diarreicas agudas em menores de 5 anos.

\section{DISCUSSÃO}

A taxa de mortalidade infantil é um dos indicadores mais utilizados para análise da situação de saúde de um país. Na mortalidade infantil, importante parcela da responsabilidade é atribuída aos serviços de saúde e de saneamento. Sabe-se que medidas sanitárias adequadas e serviços de saúde acessíveis e de boa qualidade podem ter um impacto positivo na redução da mortalidade infantil (14).

Os dados utilizados neste estudo mostraram que a mortalidade infantil na América Latina foi maior em países como a Bolívia (50,0 por 1000 nascidos vivos), a Nicarágua (33,0 por 1000 nascidos vivos) e a Guatemala (30,0 por 1000 nascidos vivos), nos quais a cobertura por serviços de esgotamento sanitário é baixa, respectivamente 25,0\%, 52,0\% e $81,0 \%$, para a população urbana. Assim, observou-se uma correlação inversamente proporcional entre a taxa de mortalidade infantil e a cobertura populacional por esgotamento sanitário nos países da América Latina. Tal resultado é corroborado por estudos anteriores (12, 13), que enfocaram os países da América Latina e do Caribe para o ano de 2002 e os estados brasileiros para o ano de 2001. Esses estudos identificaram que quanto menor a cobertura populacional por sistemas de esgotamento sanitário, maior a mortalidade infantil.

Identificou-se, ainda, que o gasto público com saúde como proporção do PIB apresentou correlação negativa com a taxa de mortalidade infantil nos países da América Latina. Segundo a Organização das Nações Unidas (15), os governos deveriam dar prioridade ao financiamento das intervenções em serviços de saúde básicos de forma a reduzir a mortalidade infantil. Para Cairncross (16), investimentos em campanhas educativas, como a promoção da lavagem das mãos com sabão, desempenham um papel central na prevenção de enfermidades que 
FIGURA 2. Regressão linear simples entre mortalidade em menores de 5 anos e cobertura por sistemas de abastecimento de água, América Latina, 2008

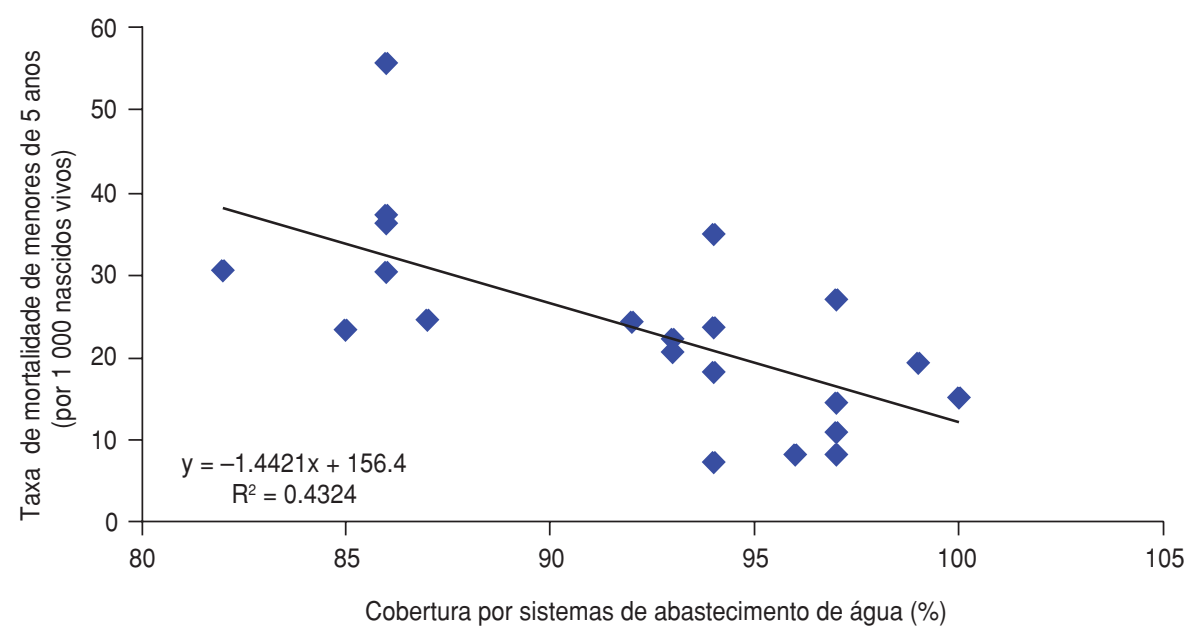

FIGURA 3. Regressão linear simples entre mortalidade proporcional por enfermidades diarreicas agudas em menores de 5 anos e taxa de crescimento anual da população, América Latina, 2008

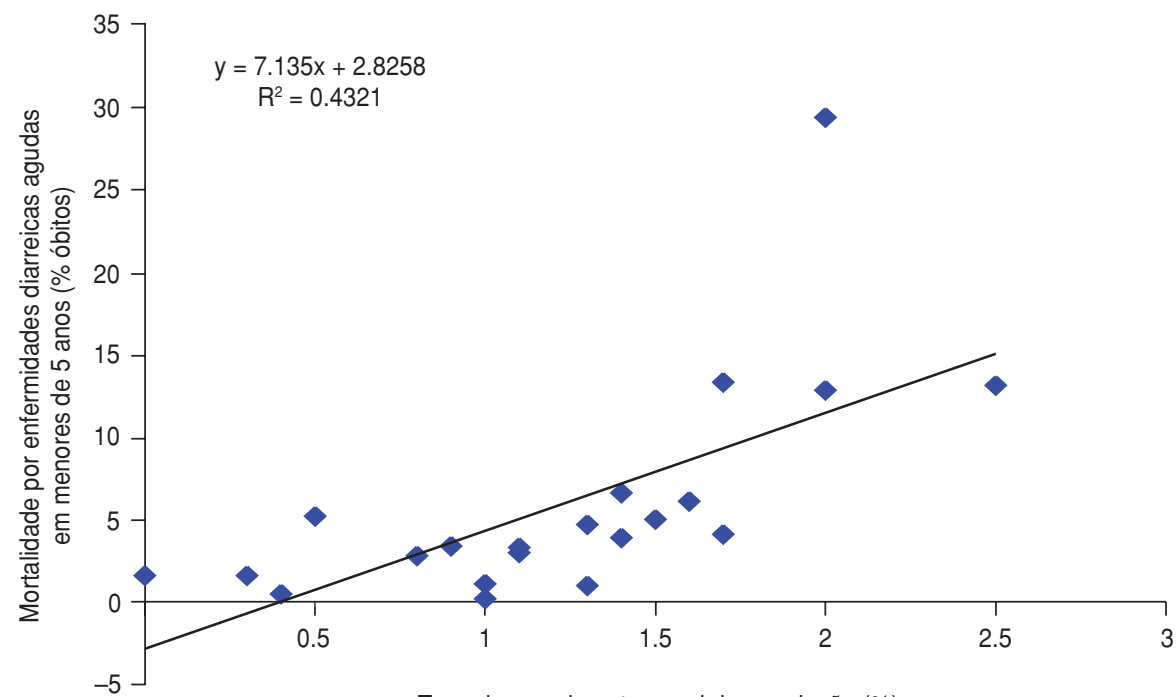

Taxa de crescimento anual da população (\%)

podem levar à mortalidade infantil, em complemento à ampliação dos serviços de saneamento.

Boing e Boing (17) afirmam que reduzir a taxa de mortalidade infantil por causas evitáveis é fundamental, mas que, entretanto, há desafios adicionais. Um deles é diminuir a desigualdade na distribuição dos óbitos entre os estratos sociais e diferentes regiões geográficas. A alocação de recursos e o desenvolvimento de estratégias sociais e de saúde devem levar em conta diferentes realidades locais. Para os autores, a compreensão do papel protagonista das condições de vida sobre a mortalidade infantil por causas evitáveis também deve permear as ações que visem à minimização da magnitude e da desigualdade dos óbitos infantis evitáveis.

Já o indicador cobertura por sistemas de abastecimento de água apresentou uma correlação inversamente proporcional à mortalidade de menores de 5 anos de idade na América Latina. De acordo com dados do Fundo das Nações Unidas para a Infância (UNICEF) (5), a água segura é uma condição necessária para melhorar a nutrição, reduzir as taxas de mortalidade materna e de menores de 5 anos e garantir a proteção contra doenças. Outro benefício da água limpa e do esgo- tamento sanitário é o fato de contribuírem para melhores taxas de frequência escolar e para um melhor aproveitamento, uma vez que as crianças não são impedidas de frequentar a escola por terem de ir buscar água para as suas famílias. Heller e Azevedo (18) afirmam que crianças com idade entre 1 e 5 anos residentes em áreas sem abastecimento de água e sem esgotamento sanitário têm uma probabilidade cinco vezes maior de apresentarem diarreia quando comparadas com crianças residentes em áreas onde esses serviços sanitários estão presentes.

Ainda, quanto à mortalidade de menores de 5 anos de idade, encontrou-se uma correlação diretamente proporcional com a razão de renda entre os $20 \%$ com maior renda e os $20 \%$ com menor renda nos países da América Latina, ou seja, com a concentração da renda. Portanto, a mortalidade de menores de 5 anos é afetada por fatores relacionados à renda. O processo latino-americano de urbanização se deu de forma desigual, com grandes diferenças entre ricos e pobres. A América Latina é considerada a região mais desigual do mundo (4), com cerca de um quarto da população das cidades vivendo em favelas ou assentamentos precários. As doenças da pobreza começam a emergir entre 1 e 5 anos de idade com mais intensidade, como já registrado por autores como Teixeira e Heller (19) e Franceschini et al. (20).

Neste estudo, encontrou-se uma correlação diretamente proporcional entre a mortalidade proporcional por enfermidades diarreicas agudas em menores de 5 anos de idade e a proporção de nascidos vivos de baixo peso ao nascer. $\mathrm{O}$ baixo peso ao nascer - menos de $2500 \mathrm{~g}$ - afetou, em média, 8,2\% das crianças na América Latina nascidas entre 2005 e 2008. A principal causa do baixo peso ao nascer em todo o mundo é a prematuridade. Contudo, as causas da prematuridade são múltiplas, algumas ainda desconhecidas, segundo Leal et al. (21). O baixo peso do bebê prematuro está relacionado à sua idade de gestação (6, 7 meses) e não significa desnutrição, mas um aumento de risco em relação a algumas doenças. Segundo Heller e Azevedo (18), a variável baixo peso ao nascer foi identificada como um dos fatores que, associados ao fato de se morar em áreas sem saneamento, aumenta a probabilidade de ocorrer infecção por protozoários nas crianças, podendo levar ao óbito por enfermida- 
des diarreicas agudas em menores de 5 anos.

Ainda, quanto à mortalidade proporcional por enfermidades diarreicas agudas em menores de 5 anos, encontrou-se uma correlação inversamente proporcional com a renda per capita. Tal achado é corroborado por Franceschini et al. (20) e por Benício e Monteiro (22), que identificaram que a mortalidade entre 1 e 4 anos é afetada por fatores relacionados à renda. A criança menor de 5 anos já não recebe os anticorpos da mãe e brinca no peridomicílio, se expondo mais ao ambiente e aumentando o risco de adquirir infecções intestinais. Há, ainda, o mecanismo da competição entre irmãos, muito mais importante em famílias pobres, onde se disputam o alimento e a atenção dos familiares. As doenças da pobreza começam a emergir nesse período com mais intensidade. Para o ano de 2010, a Comisión Económica para América

1. Heller L. Saneamento e saúde. Brasília: OPAS/OMS; 1997.

2. Daniel LA. Processos de desinfecção e desinfetantes alternativos na produção de água potável. Rio de Janeiro: Programa de Pesquisas em Saneamento Básico — PROSAB; 2001.

3. Organização Pan-Americana de Saúde (OPAS). Saúde nas Américas: 2007 - v1 Regional. Washington, DC: OPAS; 2007.

4. United Nations Human Settlements Programme (ONU-Habitat). Estado de las ciudades da América Latina y el Caribe 2010. Disponívelem:http:/ / www.regionalcentrelacundp.org/images/stories/DESCENTRALI ZACION/herramientas/estadociudades.pdf Acessado em maio de 2011.

5. Comisión Económica para América Latina y el Caribe (CEPAL). Objetivos de Desarrollo del Milenio: una mirada desde América Latina y el Caribe. Santiago: División de Desarrollo Social; 2005. Disponível em: eclac.org/ cgi-bin/getProd.asp?xml=/publicaciones / $\mathrm{xml} / 1 / 21541 /$ P21541.xml\&xsl=/tpl/p9f. xsl\&base $=/$ tpl $/$ top-bottom.xsl Acessado em dezembro de 2012.

6. UNICEF. Progress for children: a report card on water and sanitation. Nova Iorque: UNICEF; 2006.

7. Fundo das Nações Unidas para a Infância (UNICEF). Água é vida, saneamento é dignidade - Dia Mundial da Água, 22 de março de 2008. Disponível em: unicef.org/brazil/ pt/media_11765.htm Acessado em abril de 2011.

8. Fundo das Nações Unidas para a Infância (UNICEF). Situação Mundial da Infância 2011. Adolescência: uma fase de oportunidades. Disponível em: unicef.pt/18/Relatorio_ SOWC_2011.pdf Acessado em maio de 2011.

9. Rothman KJ, Greenland S, Lash TL. Modern epidemiology. $3^{\text {a }}$ ed. Philadelphia: Lippincott Willians \& Wilkins; 2008.
Latina y el Caribe (CEPAL) estimou que $32,1 \%$ dos habitantes latino-americanos estivessem em situação de pobreza e $12,9 \%$ na indigência (5).

No presente estudo, encontrou-se correlação diretamente proporcional entre a taxa de crescimento anual da população nos países da América Latina e a mortalidade proporcional por enfermidades diarreicas agudas em menores de 5 anos de idade. Tal correlação pode ser explicada pelo acentuado crescimento populacional dos países da América Latina. $\mathrm{Na}$ maioria das periferias das grandes e médias cidades da região predomina a exclusão sanitária, com crescimento urbano desordenado e falta de sustentabilidade ambiental, com ausência ou deficiência na infraestrutura sanitária, como citado por vários autores $(4,23,24)$.

Neste trabalho, duas foram as limitações principais. $\mathrm{O}$ delineamento utilizado, desenho ecológico, apresenta limi-

\section{REFERÊNCIAS}

10. United Nations Population Fund (UNFPA). World Population Prospects, the 2010 Revision. Disponível em: esa.un.org/unpd/wpp/ index.htm Acessado em maio de 2011.

11. Organização Pan-Americana da Saúde. Iniciativa regional de datos básicos en salud. Sistema generador de tablas. Disponível em: www.paho.org/Spanish/SHA/coredata/ tabulator/newTabulator.htm Acessado em julho de 2010.

12. Teixeira JC, Pungirum MEMC. Análise da associação entre saneamento e saúde nos países da América Latina e do Caribe, empregando dados secundários do banco de dados da Organização Pan-Americana de Saúde - OPAS. Rev Bras Epidemiol. 2005;8(4):365-76.

13. Teixeira JC, Guilhermino RL. Análise da associação entre saneamento e saúde nos estados brasileiros, empregando dados secundários do banco de dados Indicadores e Dados Básicos para a Saúde 2003 - IDB 2003. Eng Sanit Ambient. 2006;11(3):277-82.

14. Caldeira AP, França E, Perpétuo IHO, Goulart EMA. Evolução da mortalidade infantil por causas evitáveis, Belo Horizonte, 1984-1998. Rev Saude Publica. 2005;39(1):67-74.

15. United Nations Millennium Campaign (UNMC). Objetivos do Milénio: reduzir em dois terços a mortalidade infantil. Disponível em: http://www.un.org/millenniumgoals/ Acessado em maio de 2011.

16. Cairncross S. Handwashing with soap - a new way to prevent ARIs? Trop Med Int Health. 2003;8(8):677-9.

17. Boing AF, Boing AC. Mortalidade infantil por causas evitáveis no Brasil: um estudo ecológico no período 2000-2002. Cad Saude Publica. 2008;24(2):447-55.

18. Heller L, Azevedo EA. Exclusão sanitária em Belo Horizonte (MG): caracterização e associação com indicadores de saúde. Em: Fundação Nacional da Saúde. $1^{\circ}$ Caderno de tações referentes às técnicas de análise de dados e a vulnerabilidade à falácia ecológica; e o uso de dados secundários, cuja precisão não pode ser avaliada.

Entre as recomendações deste trabalho, pode-se afirmar que a melhoria da saúde pública na América Latina pode ser atingida pela ampliação do acesso aos serviços públicos de abastecimento de água e de esgotamento sanitário. Tal ampliação deve ser associada, entre outros, ao aumento da renda per capita na região, à ampliação da escolaridade média da população, à ampliação de campanhas de educação sanitária e à implantação de políticas públicas ligadas à atenção primária à saúde.

Agradecimentos. À Pró-Reitoria de Pesquisa da Universidade Federal de Juiz de Fora (PROPESQ) pela concessão de uma bolsa de iniciação científica. pesquisa em engenharia de saúde pública. Brasília: Funasa; 2006. Pp. 71-98.

19. Teixeira JC, Heller L. Fatores ambientais associados à diarréia infantil em áreas de assentamento subnormal em Juiz de Fora, Minas Gerais. Rev Bras Saude Mater Infant. 2005;5(4): 449-55.

20. Franceschini VLC, Rodrigues RN, Machado CJ. Análise espacial do perfil dos nascidos vivos vulneráveis ao óbito infantil por áreas de abrangência dos Centros de Saúde em Belo Horizonte, 2000. Cad Saude Colet. 2009;17(2): $333-50$.

21. Leal MC, Gama SGN, Ratto KMN, Cunha CB. Uso do índice de Kotelchuck modificado na avaliação da assistência pré-natal e sua relação com as características maternas e o peso do recém-nascido no Município do Rio de Janeiro. Cad Saude Publica. 2004;20 Suppl 1:S63-S72.

22. Benicio MHDA, Monteiro CA. Tendência secular da doença diarréica na infância na cidade de São Paulo (1984-1996). Rev Saude Publica. 2000;34(6 suppl):83-90.

23. Melli LC, Waldman EA. Temporal trends and inequality in under-5 mortality from diarrhea. J Pediatr (Rio J). 2009:85(1):21-7.

24. Pocai NL, Zanotelli CT, Felipi CC. Correlação entre as condições ambientais e óbitos de crianças menores de cinco anos de idade por diarreia aguda nas cinco regiões brasileiras. Rev Saude e Ambiente 2007:8(1):7-11.

Manuscrito recebido em 16 de novembro de 2011. Aceito em versão revisada em 12 de setembro de 2012. 
ABSTRACT Objective. To study the association between water and sanitation coverage and epidemiological indicators in Latin America, using secondary data from the Pan American Health Organization (PAHO) for the period of 2005 to 2010.

Association between sanitation services coverage and epidemiological indicators in Latin America: a study with secondary data

Methods. An ecological study was carried out including data from 21 Latin American countries, with a total estimated population of 596 million. The following variables were analyzed: infant mortality in children under 1 year in 2008, mortality in children under 5 years in 2008, and mortality from acute diarrheal diseases in children under 5 years in 2008. These indicators were analyzed by means of the "one-on-one" correlation with risk factors in five categories (demographic, socioeconomic, child health, public spending on health care, health care, and sanitation coverage).

Results. The average values for the region were 18.2 deaths per 1000 live births in children under 1 year of age, 23.5 deaths per 1000 live births for children under 5 years, and $5.8 \%$ mortality for acute diarrheal diseases in children under 5 years. Higher water and sanitation coverage was linked with lower mortality in children under 1 and under 5 years of age. Higher mortality from acute diarrheal diseases in children under 5 years was associated with higher annual population growth.

Conclusions. The improvement of public health in Latin America depends on the expansion of access to public water and sanitation systems.

Key words Public health; sanitation; water; sewage; Latin America. 